\title{
Variable-Radii Method Based on Probing Mechanism (VRPM): An Energy Conservation Method for Wireless Active Sensor Networks
}

\author{
Qi Zhou, Takuya Asaka, and Tatsuro Takahashi \\ Department of Communications and Computer Engineering, \\ Graduate School of Informatics, Kyoto University, Kyoto 606-8501, Japan \\ zhoulcube.kuee.kyoto-u.ac.jp \\ \{asaka, ttakahashi\}@i.kyoto-u.ac.jp
}

\begin{abstract}
Wireless sensor networks, which are composed of advanced MEMS (Micro-Electro-Mechanical-Systems) called sensor nodes, has been broadly researched as ad-hoc networks recently. Since sensor nodes are powered by limited-life batteries and are usually deployed in severe environments, they need to be able to conserve their power consumption as well as guarantee broad coverage and connectivity. In active sensor networks, such as RFID sensing systems, shorter sensing and communication ranges will result in a higher measuring precision and more efficient energy dissipation. In this paper, we propose a novel self-controlling method for sensor nodes to decide the most appropriate sensing radius, communication radius, and an active/sleep schedule simultaneously. The energy-saving effect is proved in our simulation section.
\end{abstract}

\section{Introduction}

A wireless sensor network is an ad-hoc network disposed to observe a certain environment, sense useful information such as temperature, humidity, pressure, light, voice, and send data efficiently to the sensor network's gateway called the BS (Base Station). The BS is connected to an IP network, such as a LAN or the internet. Users can post requirements to the sensor network base station and obtain the desired information from it.

A sensor node, which is typically composed of sensor, A/D converter, processor, memory, RF, and battery modules, is the element unit of sensor network employed to measure the surroundings. Sensor nodes usually operate in severe conditions, meaning that repair and battery exchange are difficult to do. For these special features as a network, an energy-efficient, self-scheduling method is strongly required to conserve energy by reducing energy redundancy.

There are two major types of sensors. Active sensor systems interact with the environment and observe how their actions affect the environment. Examples of active systems include RF transmitters such as RFID tag reader/writers, IR transmitters, structured lighting, and most sonar and radars. Passive sensor systems sense ambient radiation or signals. Passive systems include GPS, ambient audio, and passive motion detectors [1]. 
This study focuses on a ubiquitous RFID reader/writers networking, a typical model of active sensor networks. Countless objects with RFID tags attached are scattered across an enormous domain, and in order to identify these tags by RFID technology, large quantities of RFID reader/writers are introduced to construct a largescale sensor network. In an RFID reader/writers network, RFID reader/writers act as sensor nodes to collect tags' information using an RFID sensing system energy model, and communicate with other reader/writers or base stations to propagate data with an RF communication energy model. We will investigate the characteristic of active sensor networks to find out the dominant energy consumption fraction, and try to propose an optimal energy-aware scheduling method for active sensor networks, by varying sensing and communication radii.

The remainder of the paper is organized as follows. In the next section, we will review related work on the coverage and energy-efficiency problem briefly. Then, on the energy model, sensor nodes will be divided into several essential modules and reasonable analytic models will be abstracted in Section 3. Based on energy dissipation models, we will introduce VRPM and explain its elemental steps in Section 4. In Section 5 we use simulations to comprehensively evaluate the system's parameters and energy needs, which proves the efficiency of our proposal. Section 6 presents our conclusions and future work.

\section{Related Work}

Di Tian and colleagues proposed a solution that requires every node to know all its neighbors' positions before its schedule is determined [6]. This feature guarantees a $100 \%$ sensing coverage, but incurs a heavy communication overhead led by a priori knowledge about all neighbors. Also, this rule underestimates the area that the neighbor nodes can cover, which leads to excess energy consumption.

Fan Ye and colleagues proposed a simple localized protocol named PEAS for dynamically adjusting the schedule with a probing mechanism [5]. In this solution, after sleeping for time $T_{s}$, node wakes up and broadcasts a probing message within a certain probing range $R_{p}$ and waits for a reply. If no reply is received before a certain interval elapses, the node will transfer its status to active and remain active until the end of its battery lifetime. Although PEAS is a probabilistic method, which means full area coverage cannot be ensured, we have testified that the coverage of a monitored area is close to 1 if the threshold probing distance is less than the sensing radius.

Most recent studies on the coverage problem of sensor networks, like PEAS, assume that the sensing radius of each node is of one size. However, Fig. 1 shows the situation of covering a small domain, which implies that fixed sensing and communication radii will result in a severe redundancy problem. The redundancy we mentioned here may comprise of the following three points:

(1) It is redundant to sense the same point with multiple sensor nodes simultaneously.

(2) It is redundant to send the same sensed data multiple times due to (1).

(3) It is redundant for one node to transmit its sensed data to its neighbors within at least $2 R s$, which may be unnecessary. 
Variable-radii algorithms have been proposed recently to resolve redundancy problem caused by radius fixation. In fact, it is often assumed that a wireless device can change its transmission range to save energy [10], [11].

Jie Wu and Shuhui Yang [8] introduced a method based on one of three models. In model I, the sensing radius is fixed as maximum radius $R$. Model II adopts two sensing radii. Ideally, the count proportion of large disks to small disks is 1:2 (Fig. 2(a)). In model III, three sensing radii can be chosen by each sensor node and the count proportion here is 1:6:2 (Fig. 2(b)). Models II and III can be regarded as adopting a half-variable-radii method, which involves several discrete radii from 0 to $R$. However, due to the inequality of radius distribution (Fig. 8(a)), sensor nodes with large radii will exhaust their power much faster than those with small radii. Moreover, if there are a large proportion of small-radius sensor nodes, the involvement of too many sensor nodes will also result in energy redundancy.

Zongheng Zhou and colleagues [9] developed a series of algorithms to settle the variable-radii connected sensor coverage (VRCSC) problem. Since their goal is to select a subset of sensors with a specified sensing radius so that each point in the query region can be sensed by at least one of the selected sensors, each sensor node should know its neighbors' coordinates precisely, and a complicated calculation process has to be followed a distributed manner.
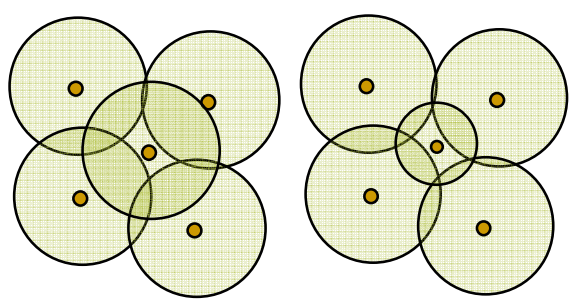

Fig. 1. Case of covering a small domain

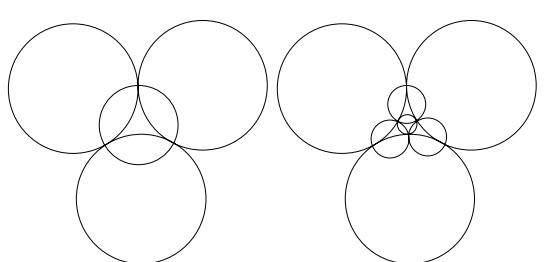

(b) Model III

Fig. 2. Discrete variable-radii model

\section{Energy Model for Active Sensor Networks}

To discuss energy efficiency, we first need to consider the energy dissipation model. In an active sensor network system, the dominant energy consumption units should be the sensing module, MCU (Micro Controlling Unit) and the wireless communication module.

As for communication module, here we consider a simple transceiver model [2]. Suppose $E_{\text {elec }}$ represents the energy of bits dissipated from the sensor in order to activate the transceiver circuitry that is present in both the receive and transmit modes, and $\varepsilon_{a m p}$ represents the energy of bits dissipated from the transmit amplifier.

In this simple radio-frequency model, to transmit $k$ bits to a distance $d$ meters assuming only path loss, the energy consumption could be:

$$
E_{T x}(k, d)=E_{\text {elec }} * k+\varepsilon_{\text {amp }} * k * d^{\beta} .
$$

To receive $k$ bits, the model of energy consumption could be: 


$$
E_{R x}(k)=E_{\text {elec }} * k
$$

In a typical wireless communication model, $\beta$ can be set to 2 .

Klaus Finkenzeller [3] and Zheng Zhu [4] introduced the model of electromagnetic radiation waves energy used in RFID reader/writers systems. As we know, some of the power will be absorbed by the targets (RFID tags), while other fractions will be scattered into different directions with different amplitude. Finally, a very small part of the power will be reflected back to the RFID reader/writers. For simplicity, we are investigating an ideal model assuming that RFID reader/writers send RF signals without any specific direction and that RF energy is scattered evenly in gradual concentric circles. We also assume that the RF energy reflected simultaneously by all tags lying in the RF reader's sensing domain, and that energy is reflected completely without any loss or absorption. Thus, for minimum energy considerations, if one RFID reader/writer scatters $\varepsilon_{a m p} k d^{\beta}$ energy, the return energy from the farthest tag it can reach is supposed to be $\varepsilon_{a m p} k d^{\beta}\left(1 / d^{2 \beta}\right)$, which indicates that the reader/writer's receive power is inversely proportional to the power of four (if $\beta=2$ ) of the distance between the tag and the reader/writer. In other words, if we double the distance, the received power at the reader/writer would be decreased to 1/16 of the previous power.

Based on the discussion above, Eq. (1) can be modified to adapt the RFID reader/writer model as:

$$
E_{T x}(k, d)=E_{\text {elec }} * k+\varepsilon_{\text {amp }} * k * d^{2 \beta} .
$$

\section{Variable-Radii Method Based on Probing Mechanism (VRPM)}

Our purpose is to propose a simple method that can be applied to distributed sensor nodes easily, without knowledge of neighbors' precise positions, while maintaining the radii distribution balance simultaneously. Although we do not try to ensure that each point of the monitored region can be covered, we will show in Section 5 that a high probabilistic coverage (very close to 1 ) can be provided.

In order to conserve energy, sensor nodes are self-configured to one of three statuses: Sleep, Active, and Probing. We propose a method for each sensor node to decide its sensing and communication radii, and Active/Sleep schedule as well. Once sensing/communication radii are decided, they will remain the same throughout the node's lifetime unless it is necessary to decide radii and schedule once more. Here are the essential steps for our VRPM method, supposing the current sensor node is named $X$. We also define $R$ and $2 R$ as maximum sensing radius and maximum communication radius respectively.

Step 1: Initially, all the sensor nodes begin with the status of sleep.

Step 2: After an exponentially distributed random time $T s$, node $X$ wakes up and broadcasts a probing message within the maximum probing range (maximum communication range) $2 R$ and waits for a reply to determine active nodes.

(a) If no reply is received within a certain interval, it will transfer its status to active. Sensing radius $R s$ is determined randomly in the range of $[0, R]$, while the communication radius is fixed as $2 R$. 
(b) If a reply is received from a certain node $A$, node $X$ will calculate the distance between $A$ and itself (denoted as $\operatorname{Dis}(A, X)$ ). If $X$ is covered by $A$, that is $\operatorname{Dis}(A, X) \leq R_{s}(A), X$ will go back to sleep. If not, that is $\operatorname{Dis}(A, X)>R_{s}(A), X$ will switch to active with a sensing radius of

$$
\alpha \times \frac{1}{n} \sum_{i=1}^{n}\left(\operatorname{Dis}\left(A_{i}, X\right)-R_{s}\left(A_{i}\right)\right),
$$

and with a communication radius of

$$
\operatorname{Max}\left(\operatorname{Dis}\left(A_{i}, X\right)\right) \text {, }
$$

where $n$ represents the count of sensor nodes on duty within $X$ 's probing range, and $\alpha$ is an overlap coefficient. $\operatorname{Max}\left(\operatorname{Dis}\left(A_{i}, X\right)\right)$ represents the maximum of all values of $\operatorname{Dis}\left(A_{i}, X\right)$.

Step 3: Once node $X$ transfers its status from sleep to active, it will remain active until the end of its battery lifetime. When $X$ is exhausted, it just dies silently and the lack of coverage will be compensated for by other sensor nodes when their next Ts comes.

Step 4: If node $X$ finds it comes to cover another active node when it tries to transfer from sleep to active status, it should instruct the covered one to sleep.

Steps 1-3 explain a basic method for each sensor node to decide when to transfer its status from sleep to active, and what the appropriate sensing and communication radii are that it should follow after its status transfer. In sensing the radius decision, $\operatorname{Dis}(A, X)-R_{s}(A)$ implies the minimum necessary radius to every single active neighbor. After the mean value is calculated, the sensing radius may still not be large enough to achieve the wide coverage desired, so overlap coefficient $\alpha$ is introduced to provide an adaptive modification. A performance evaluation for $\alpha$ will be discussed in the simulation section (Section 5).

Also, Step 4 resolves a serious problem that occurs because of the radius variation. Fig. 3 illustrates a situation supposing a radius determination sequence is nodes $A, B$, $C$, and $D$. A different consequence will arise if the determination sequence is nodes $A$, $B, D$, and $C$. It is believed that the latter one can reduce coverage redundancy, and it fits our original design better.

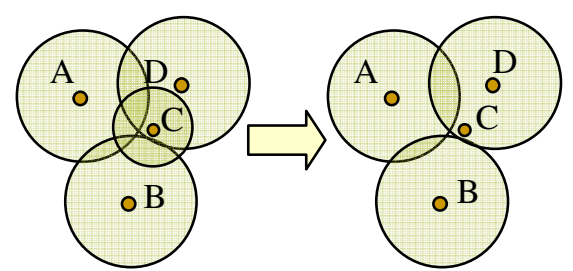

Fig. 3. Node D instructs $C$ to sleep, Step (4) of VRPM

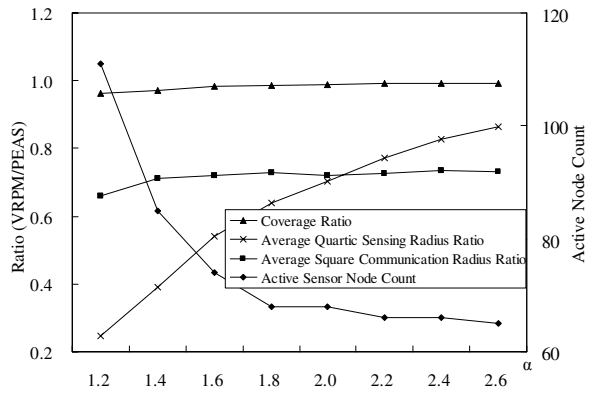

Fig. 4. Evaluation of the overlap coefficient $\alpha$ 
To ensure the connectivity of the sensor nodes, we suppose that every sensor node should have the ability to communicate with all its reachable neighbors, without considering any routing protocols. Under this rule, $\operatorname{Max}\left(\operatorname{Dis}\left(A_{i}, X\right)\right)$ is introduced to determine communication radius, which may not be the smallest efficient value. A more precise communication radius control algorithm will be studied as our future work.

\section{Performance Evaluation and Simulation}

We have programmed a simulator to prove our proposal efficient by comparing it with the conventional PEAS method. All the sensor nodes are deployed randomly to a domain measuring $100 m \times 100 m$. Every sensor node judges whether it is necessary to be on duty, and determines its appropriate sensing/communication radii with the VRPM method. We adopt a simple model that every sensor node sense $k$ bit ambient data every 1 second but only sends them to one of its neighbors every 100 seconds. That is to say, 101 seconds can be regarded as one cycle, in which every sensor node accomplishes 100 times sensing and once data propagation.

When probing, $k p$ bit is assumed to be sent. As the $k p$ is so small that communication energy dissipation can be ignored in probing status. Moreover, as the probing interval $T s$ is an exponentially distributed random time while sensor operates every one second, all the energy dissipation in probing status can be ignored if we observe for a long time enough.

Table 1. Simulation parameters

Table 2. Parameter $\beta$ settings

\begin{tabular}{l|l|l}
\hline Parameter & Sensing Unit & Communication Unit \\
\hline Maximum Radius & $10 \mathrm{~m}$ & $20 \mathrm{~m}$ \\
\hline$\beta$ & 4 & 2 \\
\hline$E_{\text {elec }}$ & $50 \mathrm{~nJ} / \mathrm{bit}$ & $50 \mathrm{~nJ} / \mathrm{bit}$ \\
\hline$\varepsilon_{\text {amp }}$ & $100 \mathrm{pJ} / \mathrm{bit} / \mathrm{m}^{4}$ & $100 \mathrm{pJ} / \mathrm{bit} / \mathrm{m}^{2}$ \\
\hline
\end{tabular}

\begin{tabular}{l|l|l}
\hline & $\beta_{s}$ & $\beta_{c}$ \\
\hline (a) & 2 & 2 \\
\hline (b) & 2 & 4 \\
\hline (c) & 4 & 2 \\
\hline (d) & 4 & 4 \\
\hline
\end{tabular}

Table 3. Energy consumption (unit: $\mathrm{mW}$ )

\begin{tabular}{l|l|l|l}
\hline Status & MCU[7] & Sensing Unit & Communication Unit \\
\hline Sleep & 0 & 0 & 0 \\
\hline Probing & 16.5 & 0 & $\left(0.05+10^{-4} R c^{2}\right) k_{p}$ \\
\hline Active (Sensing) & 16.5 & $\left(0.05+10^{-4} R s^{4}\right) k$ & 0 \\
\hline Active (Communication) & 16.5 & 0 & $\left(0.05+10^{-4} R c^{2}\right) 100 k+0.05^{*} 100 k$ \\
\hline
\end{tabular}

\subsection{Overlap Coefficient $\alpha$}

Figure 4 shows the variations of Coverage ratio $\left(\right.$ Coverage $_{\mathrm{VRPM}} /$ Coverage $_{\mathrm{PEAS}}$ ), Average quartic sensing radius ratio $\left(\mathrm{Rs}^{4} / \mathrm{R}^{4}\right)$, Average square communication radius ratio $\left(\mathrm{Rc}^{2} /(2 \mathrm{R})^{2}\right)$, and Active sensor node count at an overlap coefficient $\alpha$ range of (1.2, 2.6). As we can confirm from the chart, $\alpha$ essentially implies the criterion of $\mathrm{Rs}^{4} / \mathrm{R}^{4}$, and VRPM provides a high coverage even if $\alpha$ is much less than 1 . Also, $\mathrm{Rc}^{2} /(2 \mathrm{R})^{2}$ is a stable value because the maximum distance is adopted as the communication radius 
in our method. However, the point we should concentrate on is the trade-off between the active sensor node count and the average sensing radius.

\subsection{Energy Dissipation}

Table 1 shows the reasonable parameters used in our simulation for the energy dissipation model, and the energetic consumption volume can be formulated according to Table 3, where $R c$ and $R s$ represent the communication radius and sensing radius, respectively.

As we have mentioned in Section 3, $\beta s=4$ and $\beta c=2$ are regarded as a typical parameter set in an active sensor network, according to Table 3, one cycle's total power $P_{\text {total }}$ in the entire sensor network can be calculated with the following equation:

$$
\begin{aligned}
& P_{\text {total }} \\
= & \left(P_{\text {sensing }}+P_{\text {communication }} * N_{\text {Active }}\right. \\
= & \left\{\left\{\left[16.5+\left(0.05+10^{-4} R_{s}^{4}\right) k\right] * 100\right\}+\left\{\left[16.5+\left(0.05+10^{-4} R_{c}{ }^{2}\right) 100 k\right]\right.\right. \\
& +(16.5+0.05 * 100 k)\}\} * N_{\text {Active }},
\end{aligned}
$$

where $P_{\text {sensing }}$ and $P_{\text {communication }}$ denote power consumption by sensing and communication modules respectively, and $N_{\text {Active }}$ represents the active sensor node count. The energy dissipation due to probing mechanism can be ignored approximately because the probing traffic volumes $k_{p}$ is much less than the sensing traffic volumes $k$. In PEAS, $R s=10, R c=20, N_{\text {Acitve }}=67.2$ and

$$
P_{\text {total }}=(1683+119 k) * 67.2 \text {. }
$$

In the same way, we calculated all formulas by the variation of $\alpha$ and produced the graphs in Fig. 5(c).

The results indicate that VRPM performs well in almost all traffic volumes $k$. In particular, as the traffic grows, VRPM even manages to reduce the energy consumption by about $40 \%$ in comparison to PEAS. As another consequence, we confirmed that a too small overlap coefficient does not bring us high performance because too many sensor nodes are involved in the sensing service. On the other hand, a large overlap coefficient implies a large average sensing radius, which results in a performance close to PEAS.

For universality of the sensing and communication energy model, we varied the essential parameter $\beta$ of energy dissipation model as in Table 2, where we define $\beta_{s}$ and $\beta_{c}$ as the exponent for sensing and communication respectively, and the energy dissipation results are listed in Fig. 5 (a) to (d), where the vertical axis is in the unit of the power ratio dividing VRPM by PEAS.

Since the communication radius is much larger than the sensing radius (approximately double), the dominating partition of energy consumption ascribes to the communication module in cases (a), (b) and (d) of Fig. 5, where $\beta s \leq \beta c$. As we have concluded from Section 5.2, a variable communication radius does not cause any total power fluctuation as the overlap coefficient $\alpha$ varies, which results the parallel curves in Fig. 5(a), (b) and (d). On the other hand, VRPM reduces the total energy dissipation on most occasions, even managing to boost performance by almost $20 \%$ as $\beta$ increases. 


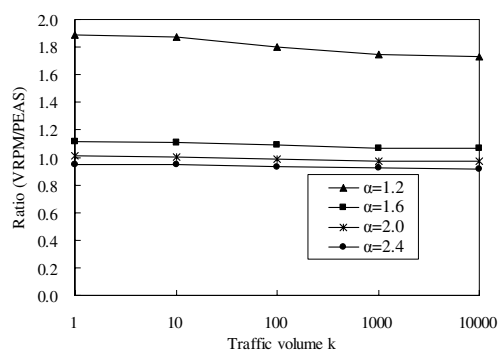

(a) Case of $\beta_{s}=2$ and $\beta_{c}=2$.

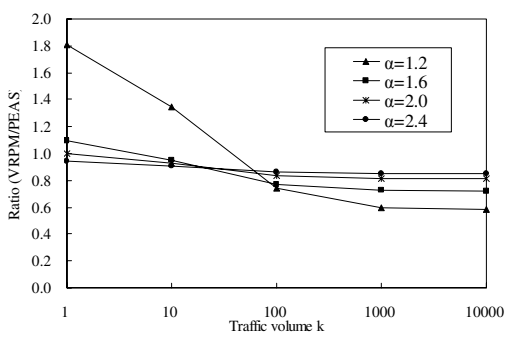

(c) Case of $\beta_{s}=4$ and $\beta_{c}=2$.

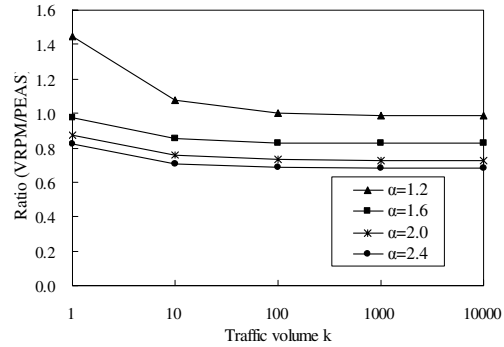

(b) Case of $\beta_{s}=2$ and $\beta_{c}=4$.

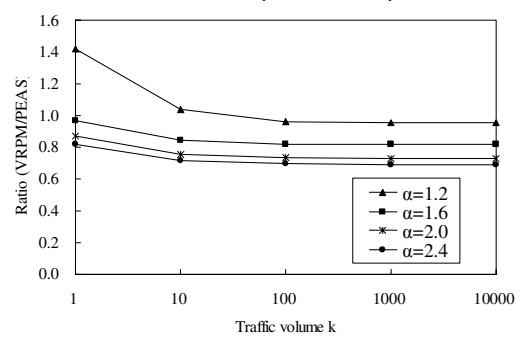

(d) Case of $\beta_{s}=4$ and $\beta_{c}=4$.

Fig. 5 . Energy dissipation evaluations by the variation of parameter $\beta$

In case (c), the sensing module comes to dominate the energy dissipation of the entire sensor network because $\beta s>\beta c$ so that the overlap coefficient $\alpha$ becomes the protagonist. Although VRPM with a small $\alpha$ value cannot exceed PEAS at a low sensing traffic volume, it decreases the energy outstandingly as long as the sensing traffic becomes large enough. This implies that local sensing with a small sensing radius will be the optimal way in instances of high sensing traffic volumes.

\subsection{Sensing/Communication Frequency}

A simple sensing/communication frequency model mentioned at the beginning of Section 5 was applied in the above discussion. However, the sensing/communication

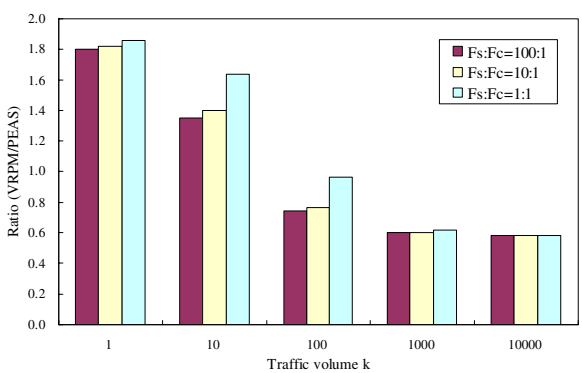

(a) Case of $\alpha=1.2$.

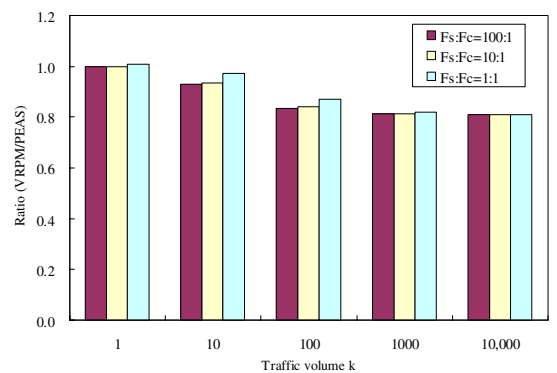

(b) Case of $\alpha=2.0$.

Fig. 6. Sensing/communication frequency evaluations 
frequency will not be a constant in practice. Therefore, we define $F s$ and $F c$ as the sensing frequency (the number of sensing events per unit time) and the communication frequency (the number of communication events per unit time), respectively, so that $F s / F c$ denotes the frequency proportion between sensing and communication. Energy dissipation is evaluated when $F s / F c$ varies as 100:1, 10:1 and 1:1 (Fig. 6).

Evident fluctuations cannot be elicited from the above charts as $F s / F c$ varies, which means that the time of sending sensed data does not affect the energy dissipation performance.

\subsection{Density of Sensor Nodes}

Until now, all discussions have been based on a high-density sensor network. We deployed 1,200 sensor nodes to the $100 m \times 100 m$ monitored region, giving a nodes density of 12 nodes $/ 100 \mathrm{~m}^{2}$. In this section, a low-density sensor network (3 nodes $\left./ 100 \mathrm{~m}^{2}\right)$ and a medium-density sensor network $\left(6\right.$ nodes $\left./ 100 \mathrm{~m}^{2}\right)$ are evaluated to judge if VRPM is density independent.

Fig. 7 explains the comparison of three different density statuses. Whatever value the overlap coefficient $\alpha$ has, VRPM provides a similar performance when the sensor node density varies from low to high, thus we can concluded that VRPM is not influenced by density.

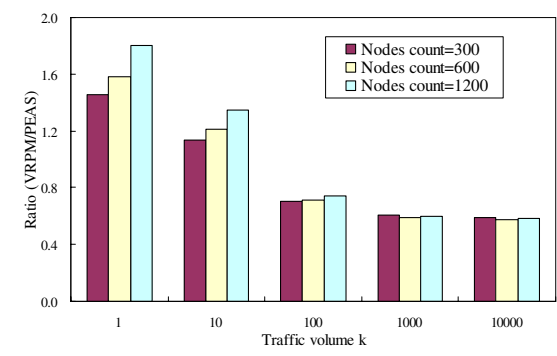

(a) case of $\alpha=1.2$.

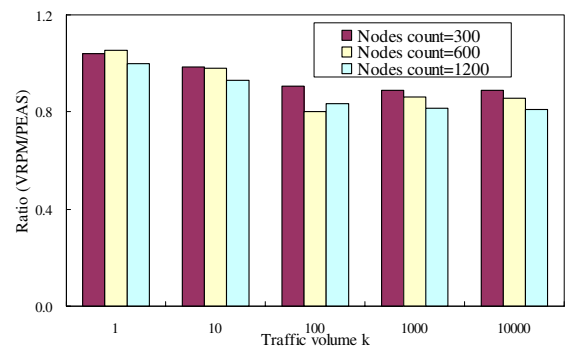

(b) Case of $\alpha=2.0$.

Fig. 7. Sensor node density evaluations

\section{Conclusion and Future Work}

The dominating energy consumption of active sensor networks lies in sensing modules and communication modules. VRPM is an efficient variable-radius energy conservation method that reduces energy redundancy in active sensor networks. The efficiency of energy conservation by VRPM was evaluated in several kinds of sensing and communication models to prove its broad applicability. Inside VRPM, an overlap coefficient $\alpha$ is designed for sensor nodes to decide their appropriate radii according to their ambient situation. A self-adaptable algorithm based on our discussions on the overlap coefficient $\alpha$ can be easily designed and embedded into every sensor node. We then proved that the energy consumption is independent of the sensing/communication frequency and density of sensor nodes through a series of simulations. Finally, we showed that VRPM can also maintain a geographical balance of 
energy dissipation distribution, thereby extending the lifetime extension of the entire sensor network.

In future work, we plan to realize and evaluate VRPM on a real Mote ${ }^{\circledR}$ system.

\section{References}

1. B. Yoshimi, and Y. Heights, "On Sensor Frameworks for Pervasive Systems", Workshop on Software Engineering for Wearable and Pervasive Computing (ICSE 2000), June 2000.

2. W. R. Heinzelman, A. Chandrakasan, and H. Balakrishnan, "Energy-Efficient Communication Protocol for Wireless Microsensor Networks", Proceedings of the 33rd International Conference on System Sciences (HICSS '00), January 2000.

3. K. Finkenzeller, "RFID Handbook: Fundamentals and Applications in Contactless Smart Cards and Identification", John Wiley \& Sons; 2 Edition, May 9, 2003.

4. Z. Zhu, "RFID Analog Front-End Design Tutorial", Auto-ID lab at University of Adelaide.

5. F. Ye, G. Zhong, J. Cheng, S. Lu, and L. Zhang, "PEAS: A Robust Energy Conserving Protocol for Long-Lived Sensor Networks," Proc. IEEE Int'l Conf. Network Protocols (ICNP 2002), IEEE CS Press, pp. 200-201, 2002.

6. D. Tian and N.D. Georganas, "A Coverage-Preserving Node Scheduling Scheme for Large Wireless Sensor Networks", Proc. 1st ACM Workshop Wireless Sensor Networks and Applications, ACM Press, pp. 32-41, 2002.

7. V. Raghunathan, C. Schurgers, S. Park, and M. B. Srivastava, "Energy-Aware Wireless Microsensor Networks", IEEE signal processing magazine, pp. 40-50, March 2002.

8. J. Wu and S. Yang, "Coverage Issue in Sensor Networks with Adjustable Ranges", ICPPW'04, August 15 - 18, 2004

9. Z. Zhou, S. Das, and Himanshu Gupta, "Variable-Radii Connected Sensor Cover in Sensor Networks", SECPM2004, October 4, 2004.

10. J. E. Wieselther, G. D. Nguyen, and A. Ephremides, "On the Construction of EnergyEfficient Broadcast and Multicast Trees in Wireless Networks", in Proceedings of the IEEE INFOCOM, 2000.

11. M. Cagalj, J. Hubaux, and C. Enz, "Minimum-energy Broadcast in All Wireless Networks: NP-completeness and Distributed Issues," in Proceedings of the International Conference on Mobile Computing and Networking (MobiCom), 2002. 